\title{
Depth Sensitive Atomic Resolution Spectroscopy and Imaging of Highly Strained YSZ/STO Epitaxial Heterostructures
}

Timothy J. Pennycook ${ }^{1,2}$, Lewys Jones ${ }^{1}$, Mariona Cabero ${ }^{3}$, Alberto Ribera-Calzada ${ }^{3}$, Carlos Leon ${ }^{3}$, Maria Varela ${ }^{4,3}$, Jacobo Santamaria ${ }^{3}$ and Peter D. Nellist ${ }^{1,2}$

1. Department of Materials, University of Oxford, Parks Road, Oxford OX1 3PH, UK

2. SuperSTEM Laboratory, Daresbury, WA4 4AD, UK

3. Grupo de Fisica de Materiales Complejos, Universidad Complutense, 28040 Madrid, Spain

4. Materials Science and Technology Division, Oak Ridge National Laboratory, Oak Ridge, Tennessee 37831, USA

Yttria-stabilized zirconia (YSZ) is one of the most commonly used electrolytes in solid oxide fuel cells because of its high $\mathrm{O}$ mobility. By growing YSZ as a thin film sandwiched between strontium titanate (STO) a remarkable increase of up to eight orders of magnitude has been achieved in its ionic conductivity near room temperatures [1]. STEM imaging showed the YSZ layers to be coherent with the STO and under a large 7\% expansive strain, while EELS linetraces showed the normalized integrated intensity of $\mathrm{Ti}$ L-edge to be higher than that of Sr M-edge at the interfaces, indicating that the STO termination layer is likely $\mathrm{TiO}_{2}$. Density functional theory molecular dynamics simulations assuming a $\mathrm{TiO}_{2}$ termination have been able to explain the very large enhancement in the ionic conductivity through a combination of lattice-mismatch strain and O-sublattice incompatibility [2]. Strain alone in bulk YSZ enhances $\mathrm{O}$ mobility at high temperatures by inducing extreme $\mathrm{O}$ disorder. In multilayer structures, $\mathrm{O}-$ sublattice incompatibility causes the same extreme disorder at room temperature. Direct imaging of the O sublattice with aberration-corrected STEM-EELS shows the ordered STO O sublattice blur out in the YSZ consistent with disorder and the $\mathrm{O} \mathrm{K}$-edge fine structure matches that simulated for the structure produced by the molecular dynamics simulations based on the $\mathrm{TiO}_{2}$ termination [3]. Another possible termination of the STO is SrO. Determining the actual termination and its consistency along the interface is of the utmost importance for understanding the transport properties.

One curious feature of coherent YSZ/STO heterostructures is the occasional appearance of STO like contrast in HAADF images of the YSZ layers. The layers are clearly highly strained from the appearance of strain contrast in medium angle ADF (MAADF) images, and HAADF images show the layers to be brighter on average than the neighboring STO consistent with YSZ, in which every cation column should appear similarly bright, but the individual columns show an alternating pattern of bright and dark columns continuing across the bright layer. The appearance of this contrast may lead one to conclude that, rather than YSZ, some sort of perovskite has formed between the STO layers.

Due to the so-called top-down effect, the clearest ADF images are formed when the probe is focused near the entrance surface. Furthermore, the depth of field in modern aberration corrected STEM instruments is only a few nanometers. These facts mean one will tend to form images in which the entrance surface provides the dominant source of fine image details, as the fine features of the material at depth will be blurred out. So if STO is at the entrance surface and YSZ is at depth one would see STO like contrast in the individual atomic columns, but they will appear on top of an out of focus image of the YSZ. Thus it seems likely regions in which we observe STO like contrast on a diffuse bright background are due to YSZ being buried under STO. 
Through focal series were performed to look for buried YSZ layers, and indeed show STO like contrast transform into YSZ like contrast at a specific depth. Since Sr, Y, and Zr are neighbours on the periodic table with atomic numbers 38,39 and 40 it is probably not possible to distinguish them from Z-contrast alone. Spectroscopy is needed, but EELS and EDX have not previously shown the depth sensitivity required here because of the large missing cone region in the optical transfer function [4]. The missing cone causes the longitudinal resolution to equal the minimum resolved characteristic transverse length scale of the object divided by the convergence angle. Without atomic resolution the longitudinal resolution will be very poor indeed as the minimum characteristic length will be the width of the layer, which for a $2 \mathrm{~nm}$ layer and the $32 \mathrm{mrad}$ convergence angle used in the present work gives a depth resolution of only $62.5 \mathrm{~nm}$, roughly three times the thickness of the film in this region.

By performing spectroscopy with transverse atomic resolution we improve the depth resolution to just a few nanometers. The first example of depth sensitive atomic resolution EELS is shown in Fig. 1. EELS spectrum imaging was performed in the same region with the probe focused at $18 \mathrm{~nm}$ into the film (Fig 1A) and at the entrance surface (Fig 1B). In Fig. 1A the HAADF image shows YSZ like contrast but the same region in Fig. 1B appears STO like. The Sr, Ti and $\mathrm{Zr}$ maps taken at the two depths are clearly different, demonstrating depth sensitivity, and show that in this region STO is on top of YSZ. The Zr map is sharply focused in part A but completely blurred out in part B, while the $\mathrm{Sr}$ and $\mathrm{Ti}$ maps appear out of focus in part A in comparison to part B. The columns resolved in the $\mathrm{Zr}$ map of Fig. 1A have a minimum transverse separation of about $0.28 \mathrm{~nm}$,

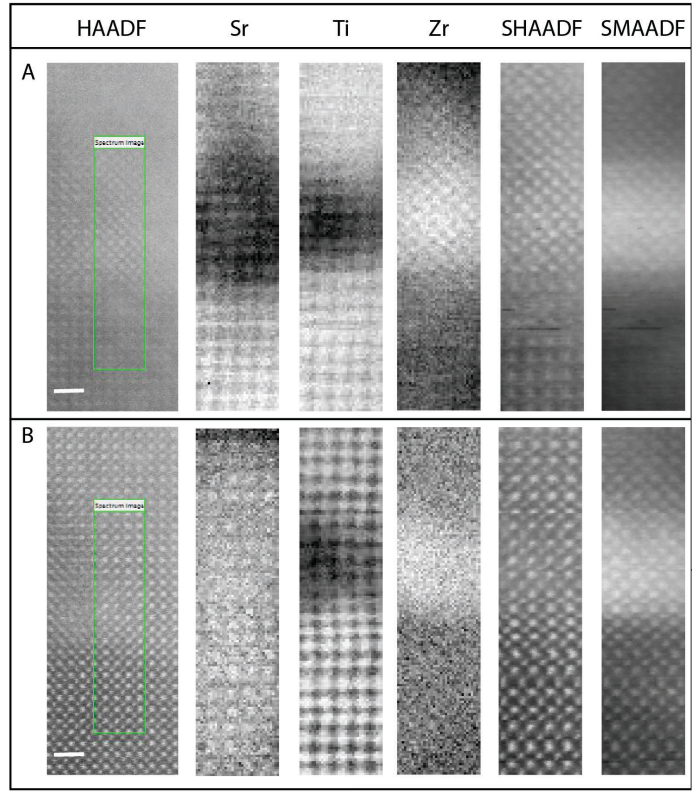
corresponding to a depth resolution of $8.75 \mathrm{~nm}$ with the 32 mrad convergence angle used. The present work demonstrates that depth sensitive atomic resolution spectroscopy is possible and indeed useful, showing that YSZ does not always grow in continuous layers even if coherent, and supporting the $\mathrm{TiO}^{2}$ termination seen in previous line scans. It also emphasizes the importance of considering that three dimensional materials may vary in all three dimensions [5]

\section{References:}

Figure 1. Depth sensitive spectrum imaging. The images shown in A were acquired with a defocus $18 \mathrm{~nm}$ below that of those in B. The first panels on the left show the HAADF survey images. The Sr (M-edge), Ti (L-edge) and $\mathrm{Zr}$ (M-edge) maps have been denoised with principle component analysis, and are shown alongside the simultaneously acquired HAADF (SHAADF) and MAADF (SMAADF). Scale bars are $1 \mathrm{~nm}$.

[1] J. Garcia-Barriocanal et al, Science 321 (2008) p. 676.

[2] T. J. Pennycook et al, Phys. Rev. Lett. 104 (2010), p. 115901.

[3] T. J. Pennycook et al, Eur. J. Appl. Phys. 54 (2010), p. 33507.

[4] G. Behan et al, Phil. Trans. R. Soc. A 367 (2009), p. 3825.

[5] This research was supported in part by the EPSRC through the UK National Facility for AberrationCorrected STEM (SuperSTEM). Research at Oak Ridge National Laboratory was sponsored by the US Department of Energy, Office of Science, Materials Sciences and Engineering Division. Research at Complutense sponsored by the European Research Council Starting Investigator Award STEMOX. 